\title{
FLASH II: Perspectives and Challenges
}

B.Faatz ${ }^{1}$, N. Baboi, V. Ayvazyan, V. Balandin, W. Decking, S. Duesterer, H.-J. Eckoldt, J. Feldhaus, N. Golubeva, K. Honkavaara, M. Koerfer, T. Laarmann, A. Leuschner, L. Lilje, T. Limberg, D. Noelle, F.

Obier, A. Petrov, E. Ploenjes, K. Rehlich, H. Schlarb, B. Schmidt, M. Schmitz, S. Schreiber, H.

Schulte-Schrepping, J. Spengler, M. Staack, F. Tavella, K. Tiedtke, M. Tischer, R. Treusch, M. Vogt, A. Willner,

Deutsches Elektronen-Synchrotron (DESY), Hamburg, Germany

J. Bahrdt, R. Follath, M. Gensch, K. Holldack, A. Meseck, R. Mitzner,

Helmholz Zentrum Berlin (HZB), Berlin, Germany

M. Drescher, V. Miltchev, J. Rönsch-Schulenburg, J. Rossbach, University Hamburg, Germany

\begin{abstract}
FLASH has been a user facility since 2005, delivering radiation in the wavelength range between 7 and $47 \mathrm{~nm}$ using the SASE principle. After the present upgrade, the wavelength range is extended to $4.45 \mathrm{~nm}$. With the 3rd harmonic accelerating module in place to linearize the longitudinal phase space, the stability and reproducibility of the machine is substantially improved. The user requests for beam time by far exceeds the time available. In order to increase user beam time and to improve the radiation properties delivered to users, a major extension of the user facility called FLASH II has been proposed by DESY in collaboration with the HZB. FLASH II is a seeded FEL in the parameter range of FLASH. As logical continuation, the seeding with HHG which started with sFLASH will result in direct seeding. Because in the foreseeable future there will probably not be HHG seed lasers available at high repetition rates down to wavelengths of $4 \mathrm{~nm}$, a cascaded HGHG scheme is proposed to produce short wavelengths.

After a first design report, the project now enters its technical design phase. During this time, the FLASH beam parameters after the present upgrade 2009/2010 will be characterized and the present design will be re-evaluated and adjusted. In addition, start-to-end simulations will complete the simulations which have been performed so far, including a design of the extraction area.
\end{abstract}

\section{Introduction}

FLASH, the free-electron laser in Hamburg, has been in operation as user facility since summer 2005 [1]. Initially, the minimum achievable wavelength was approximately $13 \mathrm{~nm}$ at an electron beam energy of $700 \mathrm{MeV}$. FLASH uses the SASE process (self-amplified spontaneous emission), a high-gain amplification in a single pass through a long undulator system. With the upgrade in 2007, the energy has been increased to $1 \mathrm{GeV}$, extending the wavelength range from $6.5 \mathrm{~nm}$ to $60 \mathrm{~nm}$ [2]. FLASH has a superconducting accelerator and operates in the burst mode. Electron bunch trains of up to $800 \mu \mathrm{s}$ in length with a repetition rate of initially $5 \mathrm{~Hz}$ have been accelerated. For users, wavelengths from $6.8 \mathrm{~nm}$ to $47 \mathrm{~nm}$ with various pulse pattern within the train have been delivered. It ranged from pulse spacings of $1 \mu \mathrm{s}$ to $20 \mu \mathrm{s}$, from single to 140 pulses. With the recent upgrade from September 2009 to February 2010, FLASH runs at $10 \mathrm{~Hz}$ with a beam energy of $1.25 \mathrm{GeV}$, and delivers now wavelengths down to $4.45 \mathrm{~nm}[3]$.

In addition to the standard SASE operation, additional undulators have been mounted into the $40 \mathrm{~m}$ long beamline section between the accelerator and the SASE undulators. The new undulators have variable gap and will be used to test the seeding scheme using an HHG source (high harmonic generation) at $38 \mathrm{~nm}$ and $13 \mathrm{~nm}$. For this purpose, a special experimental setup for a piloting experiment close to the beamline in a separate container has been set up (sFLASH) [4]. sFLASH

February 23, 2012 
is a proof-of-principle seeding experiment, and also a first step towards FLASH II. FLASH II is a second undulator beamline to be operated in parallel to the present SASE undulators. The second beamline is built into a separate tunnel followed by a new experimental hall. The undulators will all have a variable gap, the FEL process will be seeded with the HHG source and the using the HGHG (highgain harmonic generation) seeding scheme.

In this paper, we discuss the layout of the FLASH II facility, the expected beam parameters and expected performance.

\section{Extension of the FLASH Facility}

Over the past years, the FLASH facility has been steadily improved and the wavelength range extended. The number of requests for user time has been growing in time as well, as shown in Fig. 1. Extending user time by using fast switching mir-

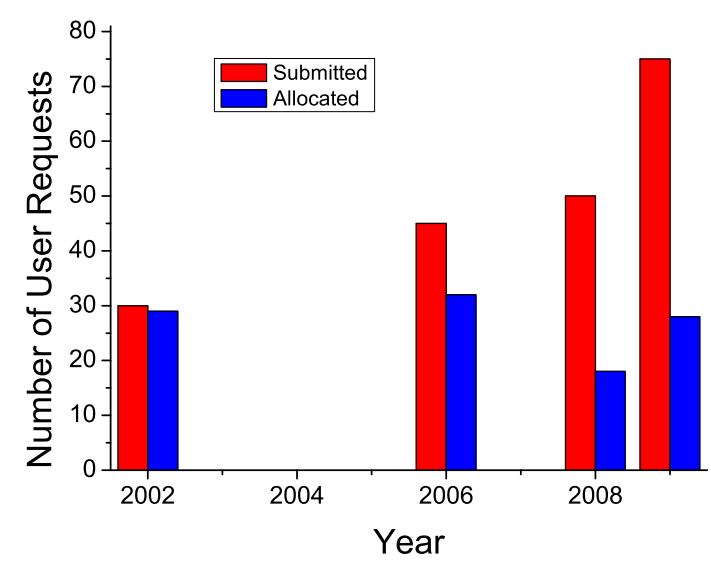

Figure 1: Beam time requested and allocated to users since the start of FLASH as user facility. The total amount of beam time available is constant but the number of requests has almost tripled. Note that the smaller number of experiments receiving beamtime in 2008 were added to the second user run which was extended by approximately 5 months.

rors and stacking experiments behind each other has already been done or is foreseen in the coming user run. However, Fig. 1 shows that this will not be enough in the future. Beside the improved quality of the radiation which is expected from the seeding scheme foreseen at FLASH II, the extension of beam time is therefore a second important argument for extending the facility with an additional undulator line.
One of the advantages of FLASH II is that it makes to large extend use of the existing facility and infrastructure. A description of the FLASH facility can be found in Ref. [5]. Behind the last accelerating module, the beam is switched between the present fixed-gap undulator line of FLASH (now referred to as FLASH I) and the new variable gap undulator FLASH II (see Fig. 2). The modification needed to the present facility is minor. In addition, the new undulator line is located in a new tunnel. This ensures that enough space is available for future upgrades and extensions and that construction can take place with minimum interference to FLASH I user operation. Only when the connection of the tunnels and of the vacuum systems is made, a shutdown is needed.

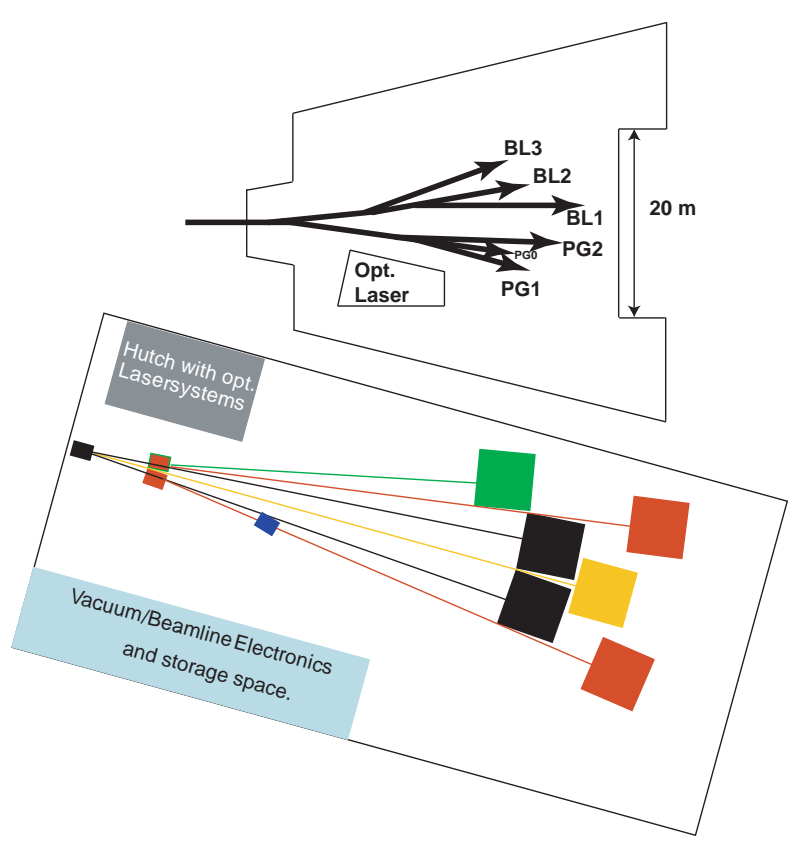

Figure 3: Present Layout of the new experimental hall. For comparison, the hall for FLASH I is also shown.

In the new tunnel, the beamline consists of a matching and diagnostics section, a seeding section which is reserved for the cascaded HGHG undulators, and a large undulator section which is used as the last amplification stage for HGHG or for direct seeding with an HHG source. The length is sufficient to allow for saturation from noise in SASE mode over the entire wavelength range. Behind this undulator section, space is reserved for an afterburner and more diagnostics. Behind the dump magnet, some $20 \mathrm{~m}$ of space is available for photon diagnostics, similar to what has been in use 


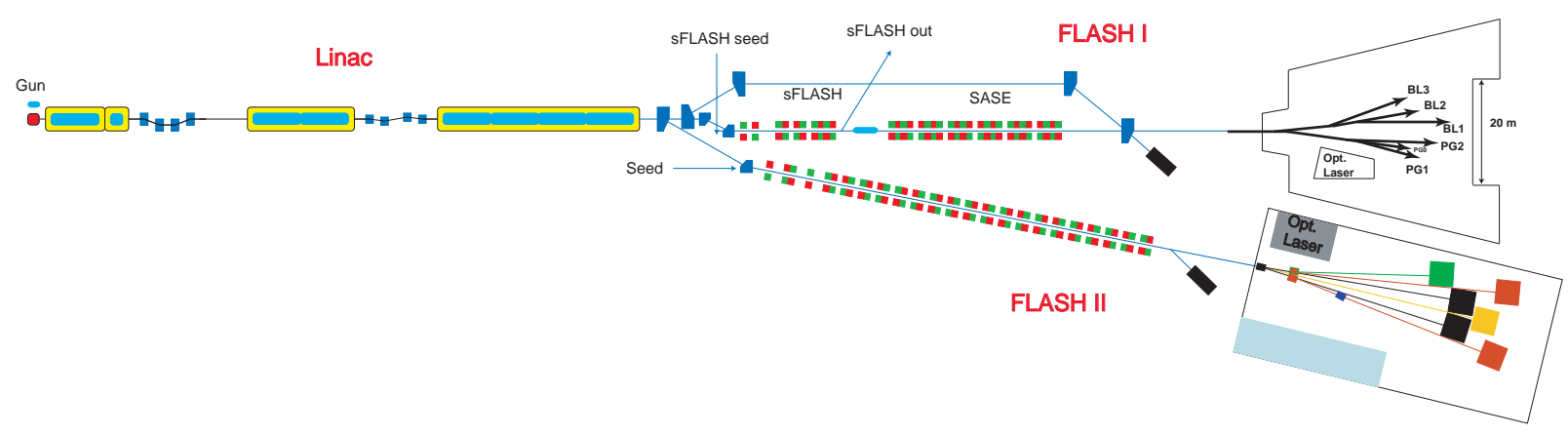

Figure 2: Schematic layout of the FLASH facility. The electron gun is on the left, the experimental hall on the right. Behind the last accelerating module, the beam is switched between FLASH I, which is the present undulator line, and FLASH II, which is the upgrade. Behind the extraction point, space is reserved for an additional laser system for seeding.

so far for FLASH [6], but extended with an on-line spectrometer [7].

Space for a total of at least five experimental stations is foreseen, not including the possibility of experiments in a row or experiments at larger angles for the longer wavelengths (indicated in green at the top of the new experimental hall in Fig. 2).

\section{FLASH II parameters and characteristics}

Table 1: Expected electron beam parameters and the properties of the final undulator for FLASH II.

\begin{tabular}{|l|c|}
\hline Electron Beam & Value \\
\hline Energy Range & $0.5-1.25 \mathrm{GeV}$ \\
Peak Current & $2.5 \mathrm{kA}$ \\
Normalized Emittance & $1.4 \mathrm{~mm} \mathrm{mrad}$ \\
Energy Spread & $0.5 \mathrm{MeV}$ \\
Average $\beta$-function & $6 \mathrm{~m}$ \\
Rep. rate & $10 \mathrm{~Hz}$ \\
Bunch separation & $1-50 \mu \mathrm{s}$ \\
\hline Undulator & Value \\
\hline Period & $\approx 31 \mathrm{~mm}$ \\
K & $\approx 0.5-2$ \\
Segment length & $2 \mathrm{~m}$ \\
Number of segments & 12 \\
\hline
\end{tabular}

In Table 1 and Table 2, the parameters expected for FLASH II are shown. Several parameters, such as the undulator period and gap of the vacuum chamber are still under discussion and may undergo minor changes. They are similar to those for FLASH I with the exception of the energy spread, which grows due to coherent synchrotron radiation in the extraction area.

\subsection{Seeding Schemes}

A cascaded HGHG is foreseen for wavelengths down to $\approx 4 \mathrm{~nm}$. The seed laser is a Ti:Sa laser at a repetition rate of $100 \mathrm{kHz}$, which is under development at DESY [8]. After frequency up-conversion, this seed enters at the beginning of the vacuum pipe, as indicated in Fig. 2. Foreseen are two frequency multiplication stages with a fresh-bunch chicane between the first radiator and second modulator. In both stages, up to the fifth harmonic is foreseen, resulting in a smallest wavelength of $8 \mathrm{~nm}$. In order to reach a shorter wavelength, the use of the seventh harmonic is possible, although only at reduced power due to the limited modulation depth compared to the energy spread. More elaborated modulator schemes allow for even higher harmonics in the HGHG stages [9]. Simulation studies especially for shorter wavelengths are ongoing. At the moment, the undulators have an approximate length of $2 \mathrm{~m}$ for the first modulator, $4 \mathrm{~m}$ of the first radiator and again $2 \mathrm{~m}$ for the second modulator. The exact period length for the different stages is part of the present optimization process.

Direct seeding with an HHG source is only foreseen for wavelengths between $10 \mathrm{~nm}$ and $40 \mathrm{~nm}$. It uses the same laser as is used for the HGHG scheme. For longer wavelengths, although not excluded, we expect problems transporting the seed to the entrance of the undulator. To ensure that the radiation source for the users is at a fixed position, the upstream undulator gaps are opened, which means that the seed laser has to reach further into the vacuum pipe at longer wavelength. For long wavelengths, SASE has to be used. At the smaller wavelength end, down to $10 \mathrm{~nm}$, the seed power expected is still enough for sufficient seeding. 
Table 2: Expected output parameters for FLASH II.

\begin{tabular}{|l|c|c|c|}
\hline Photon Beam & HHG & HGHG & SASE \\
\hline Wavelength range (fundamental) & $10-40 \mathrm{~nm}$ & $4-30 \mathrm{~nm}$ & $4-80 \mathrm{~nm}$ \\
Average single pulse energy & $1-50 \mu \mathrm{J}$ & $30-200 \mu \mathrm{J}$ & $1-1000 \mu \mathrm{J}$ \\
Pulse duration (FWHM) & $<15 \mathrm{fs}$ & $15-80 \mathrm{fs}$ & $10-200 \mathrm{fs}$ \\
Peak power (from av.) & $1-5 \mathrm{GW}$ & $1-6 \mathrm{GW}$ & $1-5 \mathrm{GW}$ \\
Spectral width (FWHM) & $\approx 0.1-1 \%$ & $\approx 0.1-1 \%$ & $\approx 0.1-1 \%$ \\
Peak Brilliance & $10^{28}-10^{31} \mathrm{~B}^{*}$ & $10^{28}-10^{32} \mathrm{~B}^{*}$ & $10^{28}-10^{31} \mathrm{~B}^{*}$ \\
\hline
\end{tabular}

* $\mathrm{B}=$ photons $/\left(\mathrm{s} \mathrm{mrad}^{2} \mathrm{~mm}^{2}(0.1 \% \mathrm{bw})\right)$

Further decrease in wavelength is possible by employing a classical HGHG scheme, where we go to a higher harmonic before saturation, thus avoiding a large energy spread which decreases the saturation power. For this reason, the HHG seed enters upstream of the first HGHG radiator (not shown in Fig. 2), which allows for a single frequency multiplication step.

A study to extend the wavelength range down to $2 \mathrm{~nm}$ is in progress. The idea is to use a short afterburner optimized for this short wavelength at an energy of $1.25 \mathrm{GeV}$. In order to allow a variable polarization of the radiation pulses, this afterburner will be an APPLE III undulator. However, this wavelength can only be reached at reduced the power, typically a few percent of the fundamental.

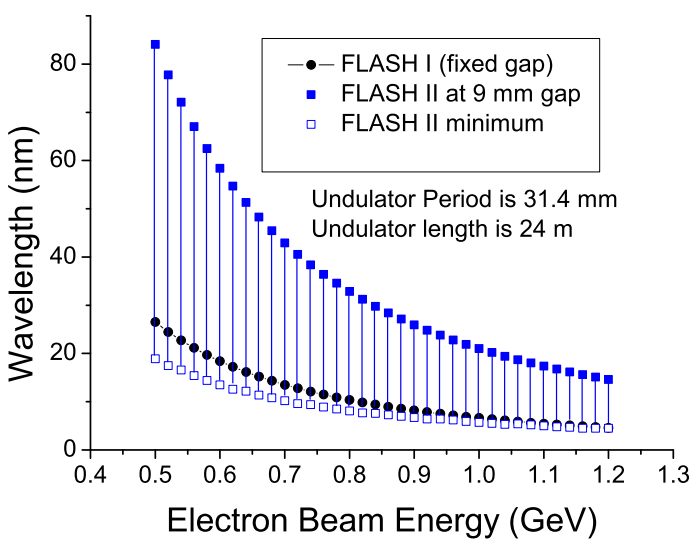

Figure 4: Tunability range of FLASH II for a given energy determined by FLASH I users. For this example, an undulator period of $31.4 \mathrm{~mm}$ has been used.
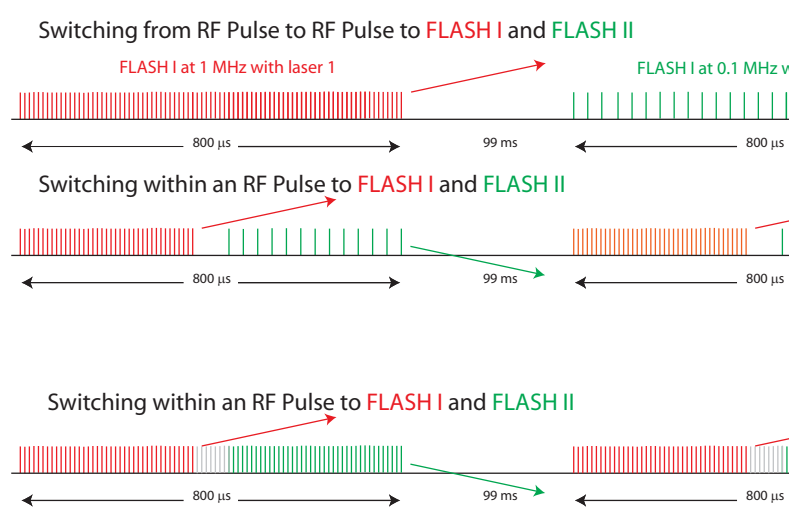

Figure 5: Switching schemes between FLASH I and FLASH II as they are at the moment considered. One can switch slowly using either one or two injection lasers (top graph) delivering $5 \mathrm{~Hz}$ to both users. Fast switching within one pulse train is discussed using different repetition rates with two injector laser systems (middle graph) or at one repetition rate with one laser system (bottom graph).

\subsection{Switchyard FLASH I-FLASH II}

An important issue is the way in which FLASH I and FLASH II will share the electron beam. Because FLASH I has a fixed-gap undulator, the wavelength needed here fixes the energy. Within a certain wavelength range, the FLASH II undulator gap is changed to deliver the wavelength required by users of this undulator line, as shown in Fig. 4.

A slow switching mode is foreseen in which both users get bunches at a repetition rate of $5 \mathrm{~Hz}$, while the machine is running at $10 \mathrm{~Hz}$. In FLASH the RFpulses for the acceleration of the electron beam are long $(800 \mu \mathrm{s})$. An alternative to the slow switching scheme is to make use of this: the bunch train is divided into two bursts, one goes straight to FLASH I, the second part is kicked to FLASH II - delivering beam for both lines with $10 \mathrm{~Hz}$ repetition rate each (see Fig. 5). In order to make this work, the two bursts in the pulse train need to be separated by a 
couple of microseconds. One possibility is to use a single laser system running a full burst. Different pulse pattern are produced by disturbing the electron beam orbit with a fast kicker system. The disturbed pulses would go through the undulators, but would not participate in the lasing process. Since for this option, there is no gap in the pulse train, the gray pulses in Fig. 5 are not kicked right and would be dumped in an intermediate beam dump. First promising tests of disturbing bunches with a fast kicker have been performed at FLASH.

\section{Photon Diagnostics and Transport}

Characterization of the radiation properties is important both during machine setup and user operation. As compared to FELs driven by a normal conducting accelerator, the high repetition rates of FLASH are an additional challenge. For each of the FEL pulses, which can add up to 8000 per second, one needs to know the intensity, position and angle, and the spectrum. A single shot measurement of the spectrum is especially important in SASE mode, when the spectrum will fluctuate from shot to shot. From the experience gained in FLASH I operation, we foresee gas monitor detectors to measure the absolute single pulse energy, the spectrum, and the position of the FEL pulses $[6,7]$. In addition, a Ce:YAG crystal can be placed into the beam providing a visual transverse profile of the radiation pulses. This is useful for setting up and tuning.

Many of these detectors will be available at least twice on order to distinguish between machine performance and the information for users. Beam properties may be altered by the users, for instance by using an attenuator or apertures.

In its original design, the mirror material and angle foreseen for FLASH II were copies of the layout of FLASH I, namely an angle of 2 degrees (or deflection by 4 degrees). However, this would strongly suppress the $3^{\text {rd }}$ and $5^{\text {th }}$ harmonics at the shorter wavelengths and therefore limit the applicability of FLASH. Therefore, the angle of the first deflecting mirror has been reduced to 1 degree. For the experimental hall, the angles are reconsidered and an iteration to optimize separation between experiments and availability of wavelength range has started.

\section{Summary and Outlook}

The FLASH II project is at the moment in its technical design phase. Main effort will be the

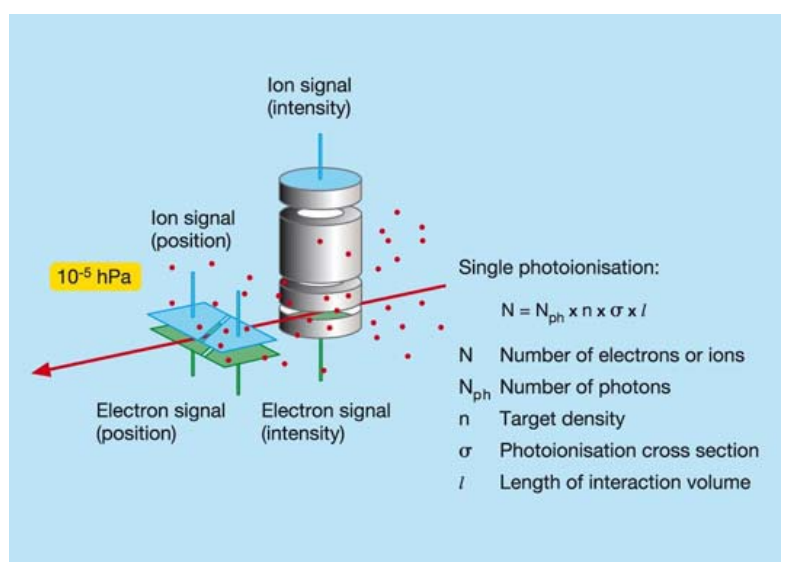

Figure 6: Schematic layout of the gas monitor detector as used at FLASH.

extraction area which is needed to maintain the good beam quality in the presence of coherent synchrotron radiation. First simulations show that even with an extraction angle larger than 10 degrees, the (projected) emittance growth can be kept below $50 \%$. In addition to ongoing simulation work, part of the study time at FLASH during the next two years will be dedicated to get operational experience with long pulse trains, needed to operate FLASH I and FLASH II simultaneously.

Delivering also variable polarization at the fundamental wavelength at full power is not part of the FLASH II proposal. However, discussions have started to make this available shortly after.

\section{References}

[1] V. Ayvazyan, et al., First operation of a free-electron laser generating GW power radiation at $32 \mathrm{~nm}$ wavelength, EUROPEAN PHYSICAL JOURNAL D (37) 2 , 297-303, 2006

[2] W. Ackermann, et al., Operation of a free-electron laser from the extreme ultraviolet to the water window, NATURE PHOTONICS 1, 336-342, 2007

[3] K. Honkavaara et.al, UPGRADE OF THE FEL USER FACILITY FLASH, presented at the IPAC10, May 2328, 2010, Kyoto, Japan.

[4] Hossein Delsim-Hashemi et.al., Status of sFLASH, the seeding Experiment at FLASH, presented at the IPAC10, May 23-28, 2010, Kyoto, Japan.

[5] S. Schreiber et.al, FEL USER FACILITY FLASH, presented at the IPAC10, May 23-28, 2010, Kyoto, Japan.

[6] K. Tiedtke et.al, The soft x-ray free-electron laser FLASH at DESY: beamlines, diagnostics and endstations, NEW JOURNAL OF PHYSICS 11, 023029

[7] P. Juranic, S. Bonfigt, M. Ilchen, K. Tiedtke, J. Viefhaus, L. Jahn, S. Klumpp, M. Martins, The new online Photoionization Spectrometer at FLASH, presented at the IPAC10, May 23-28, 2010, Kyoto, Japan. 
[8] A. Willner et.al, High Repetition Rate seeding of a FreeElectron Laser at DESY Hamburg, presented at the IPAC10, May 23-28, 2010, Kyoto, Japan.

[9] E. Allaria and G. De Ninno, Soft-X-Ray Coherent Radiation Using a Single-Cascade Free Electron Laser, Phys. Rev. Lett. 99, 014801 (2007) 